\title{
Interactive comment on "Soil organic carbon stocks are systematically overestimated by misuse of the parameters bulk density and stone content” by Christopher Poeplau et al.
}

Christopher Poeplau et al.

christopher.poeplau@thuenen.de

Received and published: 18 January 2017

We are grateful for the comments of the reviewer which were very positive and constructive and hope that we were able to improve the manuscript.

GENERAL COMMENTS Since soils represent the largest carbon reservoir of the terrestrial ecosystems, its correct estimation is essential to model the interactions between the pedosphere and the vegetation and predict the effects of climate change on ecosystems. The manuscript addresses an important topic which surely falls within the scopes of SOIL. The problems highlighted by the authors are not new to the scientific community however, probably for the first time, different methods of soil carbon stock calculation were compared by application to a common dataset therefore allowing to 
quantify the bias introduced by each of them. The manuscript is well structured, objectives are clear and methods are sound. The results are well supported by the data. I SOILD therefore recommend acceptance after some minor corrections are made.

SPECIFIC COMMENTS Add something about soil classification, to which soil types do the soils belong to? Since you have 2350 sites, give at least some general information on more common soil types and parent material.

Interactive comment

Response: We added the following sentence: "The most common soil types sampled were cambisols (24\%), anthrosols (16\%), stagnosols (13\%) and albeluvisols (11\%) and the parent material was at $93 \%$ of all sites loose sediments of varying origins."

Why did you choose to select the soils with a SOC $<8.7 \%$ ? Or is this this $8.7 \%$ the maximum SOC value of the selected plots?

Response: $8.7 \%$ SOC (or $15 \%$ SOM) is in the German soil classification the upper boarder for pure mineral soils. Organic soils, or such in transition between organic and mineral soils were excluded. We now added a citation in the respective sentence. We changed the sentence as follows: "Here, we excluded soils with a SOC content $>8.7 \%$, which are not considered mineral soils anymore (Ad-Hoc-Ag Boden, 2005), giving a total of 2350 sites and 11,514 soil samples."

Table S1. Try to sort the data and indicate the land use type: Forest, Cropland, Grassland. Based on this classification, you can then derive if the overestimation of soil $\mathrm{C}$ stock was prevalent in a certain land use type. Maybe one of the four calculation methods was used more frequently in a certain land use type? Check if this is feasible.

Response: We have done such a land use comparison and added another table in the supplement. We also added the following sentences: "Cropland was the land-use type in which stones were most often completely ignored. Eighty five percent of all reviewed cropland studies used M1 to calculate SOC stocks (Table S2). In contrast, $71 \%$ of all studies that used M4 were conducted in forest soils. This might be related to the fact, 
that stones are more abundant in forest soils and that SOC investigations in cropland soils are often restricted to the surface layer. However, only $12 \%$ of all forest studies SOILD used method M4, while M1 was the most often applied (42\%)."

lines 107-108: why statistical analyses were not conducted? I'm not sure I understood, rephrase the sentence or explain in a different way. Or delete it if not pertinent with the rest of the manuscript.

Response: Statistics is used to separate random deviations from systematic deviations. If only systematic deviations occur, no statistics are needed. We changed the section, which now reads as follows: "Due to the fact that method-induced deviations were systematic, we did not conduct statistics. As soon as the stone content is not 0 , there is always a significant difference between calculation methods, no matter how small the differences between methods would be."

\section{TECHNICAL CORRECTIONS}

*line 70: delete "where identified".

Response: We changed this accordingly.

*line 79: list the terms in the order they appear in the equation. i.e. SOC stocki, SOC con fine soil, etc.

Response: We changed the sentence as follows: "where ãĂÜBDãĂŮ_sample is the bulk density of the total sample, ãĂÛmassãĂŮ_sample is the total mass of the sample, ãĂÚvolumeãĂŮ_sample is the total volume of the sample, ãĂÚSOCstockãĂŮ_i is the SOC stock of the investigated soil layer (i) [Mg ha-1], ãÂUSSOCconãĂŮ_(fine soil) is the content of SOC in the fine soil [\%] and ãÂUdepthã soil layer [cm]."

*lines 95-101: I would suggest not to repeat the equations which were already reported in the page before, but to cite them instead. 
Response: We changed this accordingly.

*Figure 1: add a top x axis title "Volumetric stone classes"

SOILD

Response: We added the title "Volumetric stone content classes".

Interactive comment on SOIL Discuss., doi:10.5194/soil-2016-78, 2016.

Interactive

comment 